\title{
Externally pumped millimeter-wave Josephson-junction parametric amplifier
}

\author{
Levinsen, M.T; Pedersen, Niels Falsig; Sørensen, Ole; Dueholm, Benny; Mygind, Jesper
}

Published in:

I E E E Transactions on Electron Devices

Link to article, DOI:

10.1109/T-ED. 1980.20130

Publication date:

1980

Document Version

Publisher's PDF, also known as Version of record

Link back to DTU Orbit

Citation $(A P A)$ :

Levinsen, M. T., Pedersen, N. F., Sørensen, O., Dueholm, B., \& Mygind, J. (1980). Externally pumped millimeter-wave Josephson-junction parametric amplifier. I E E E Transactions on Electron Devices, 27(10), 1928-1934. https://doi.org/10.1109/T-ED.1980.20130

\section{General rights}

Copyright and moral rights for the publications made accessible in the public portal are retained by the authors and/or other copyright owners and it is a condition of accessing publications that users recognise and abide by the legal requirements associated with these rights.

- Users may download and print one copy of any publication from the public portal for the purpose of private study or research.

- You may not further distribute the material or use it for any profit-making activity or commercial gain

- You may freely distribute the URL identifying the publication in the public portal 


\section{ACKNOWLEDGMENT}

The author would like to thank A. R. Kerr and J. H. Claassen for many fruitful discussions during the course of the work.

\section{REFERENCES}

[1] Y. Taur, J. H. Claassen, and P. L. Richards, "Conversion gain in a Josephson effect mixer," Appl. Phys. Lett., vol. 24, pp. 101103, Jan. 1974.

[2] J. H. Claassen and P. L. Richards," Point-contact Josephson mixers at $130 \mathrm{GHz}$," J. Appl. Phys., vol. 49, pp. 4130-4140, July 1978.

[3] Y. Taur and A. R. Kerr, "Low-noise Josephson mixers at $115 \mathrm{GHz}$ using recyclable point contacts," Appl. Phys. Lett., vol. 32, pp. 775-777, June 1978.

[4] J. H. Claassen, Y. Taur, and P. L. Richards, "Noise in Josephson point contacts with and without RF bias," Appl. Phys. Lett., vol. 25 , pp. $759 \sim 61$, Dec. 1974 .

[5] Y. Taur, "Noise down-conversion in a pumped Josephson junction," J. Phys., vol. 39-C6, pp. 575-576, Aug. 1978.

[6] D. N. Held and A. R. Kerr, "Conversion loss and noise of microwave and millimeter-wave mixers: Part 1-Theory," IEEE Trans. Microwave Theory Tech., vol. MTT-26, pp. 49-55, Feb. 1978.

[7] F. Auracher and T. Van Duzer, "Numerical calculations of mixing with superconducting weak links," in Proc. Appl. Superconduc- tivity Conf., pp. 603-607, Sept. 1972.

[8] Y. Taur, "Josephson junctions as microwave heterodyne detectors," Ph.D dissertation, University of California, Berkeley, 1974.

[9] Y. Taur, J. H. Claassen, and P. L. Richards, "Conversion gain and noise in a Josephson mixer," Rev. Phys. Appl., vol. 9, pp. 263268 , Jan. 1974.

[10] J. H. Claassen and P. L. Richards, "Performance limits of a Josephson-junction mixer," J. Appl. Phys., vol. 49, pp. 41174129, July 1978.

[11] Y. Taur, P. L. Richards, and F. Auracher, "Application of the shunted junction model to point-contact Josephson junctions," in Proc. 13th Conf. Low Temp. Phys., vol. 3, pp. 276-280, Aug. 1972.

[12] B. D. Josephson, "Possible new effects in superconductive tunneling," Phys. Lett., vol. 1, pp. 251-253, July 1962.

[13] A. R. Kerr, private communication.

[14] F. Auracher and T. Van Duzer, "RF impedance of superconducting weak links," J. Appl. Phys., vol. 44, pp. 848-851, Feb. 1973.

[15] H. C. Torrey and C. A. Whitmer, Crystal Rectifiers. New York: McGraw-Hill Book, 1948, ch. 5.

[16] A. Van der Ziel, Noise; Sources, Characterization, Measurement. Englewood Cliffs, NJ: Prentice-Hall, 1970, ch. 2.

[17] D. W. Peterson and Y. Taur, to be published.

[18] D. W. Peterson, "The unclamped and current clamped SUPARAMP: Studies of the unbiased Josephson junction parametric amplifier," Ph.D dissertation, University of California, Berkeley, 1978.

\title{
Externally Pumped Millimeter-Wave Josephson-Junction Parametric Amplifier
}

\author{
M. T. LEVINSEN, NIELS F. PEDERSEN, OLE H. SOERENSEN, BENNY DUEHOLM, AND JESPER MYGIND
}

\begin{abstract}
A unified theory of the singly and doubly degenerate Josephson-junction parametric amplifier is presented. Experiments with single junctions on both amplifier modes at frequencies 10,35 , and 70 $\mathrm{GHz}$ are discussed. Low-noise temperature $(\sim 100 \mathrm{~K}$, single sideband (SSB)) and reasonable gain $(\sim 8 \mathrm{~dB})$ were obtained at $35 \mathrm{GHz}$ in the singly degenerate mode. On the basis of the theory and experiments, a general procedure for optimizing junction parameters is discussed and illustrated by the specific design of a $100-\mathrm{GHz}$ amplifier.
\end{abstract}

Manuscript received February 5, 1980; revised June 16, 1980. This work was supported in part by the Danish Natural Science Research Council under Grants 511-5616, 511-8527, 511-10098, 511-10279, 511-15159, and 511-20013.

M. T. Levinsen is with Physics Laboratory I, H. C. $\emptyset$ rsted Institute, DK-2100 Copenhagen, Denmark.

N. F. Pedersen, O. H. Soerensen, B. Dueholm, and J. Mygind are with Physics Laboratory I, Technical University of Denmark, DK-2800 Lyngby, Denmark.

\section{INTRODUCTION}

$P$ ARAMETRIC AMPLIFICATION based on external modulation of the nonlinear Josephson inductance has been studied for almost a decade [1]-[14]. The efforts have been devoted mainly to two different single-idler modes. The doubly degenerate amplifier (DDA) first suggested by Parrish et al. [1] uses a single matching circuit centered at the pump frequency $f_{p}$. The dominant idler is at the frequency $f_{i}=2 f_{p}$ $f_{s}$, where $f_{s}$ is the signal frequency. In the DDA maximum gain occurs at $f_{s} \simeq f_{i} \simeq f_{p}$. It has been standard to operate the DDA at zero dc-bias current where the nonlinearity is of second order (the leading term of the Josephson inductance is at $2 f_{p}$ ). The doubly degenerate mode has been thoroughly studied at $X$ band by Feldman et al. [2] , and by Wahlsten et al. [3] , and at $K a$ band by Taur and Richards [4], and Goodall et al. [5] . 
The singly degenerate amplifier (SDA) mode was suggested by Pedersen et al. [6] and later put into operation by Mygind et al. [7], [8]. The SDA employs two independent matching circuits, one for the pump, and a common signal and idler circuit centered at half the pump frequency. Here, the important idler is at $f_{i}=f_{p}-f_{s}$ and maximum gain is at $f_{s} \simeq$ $f_{i} \simeq \frac{1}{2} f_{p}$. In order to generate the idler at $f_{p}-f_{s}$, it is necessary to operate the amplifier at a finite value of dc-bias current.

In both modes, the Josephson inductance has a nonzero time average. In order to maximize the modulation depth the average value must be compensated. This may be accomplished in two ways; either by taking advantage of the intrinsic shunt capacitance of the junction and tune the $L C$ resonance (the plasma resonance) to the signal frequency by means of the dc-bias current, or, if the capacitance is negligible, to suppress the average inductance by applying a dc magnetic field and/or by means of the pump power. The latter method is most often used for the DDA mode, whereas the dc-bias tuning is more convenient with the SDA. The pump power required for the DDA may be one or two orders of magnitude higher than for the SDA. In both cases, however, the pump power required is several orders of magnitude less than for conventional varactor amplifiers.

Theoretically, both modes have an upper cutoff frequency, which for commonly used materials is in the range 250-600 $\mathrm{GHz}$. The amplifier bandwidth may be limited by the width of the internal plasma resonance or by the bandwidth of the external matching circuit. The width of the plasma resonance is typically $\Delta \omega / \omega \lesssim 0.1$. In most cases, however, the observed bandwidth is $\Delta \omega / \omega \sim 10^{-3}$, which clearly demonstrates that the embedding microwave circuit is the bandwidth-limiting factor.

In this paper we present measurements on both DDA's and SDA's. The paper is organized as follows: In Section II, the amplifier theory is briefly reviewed using a formulation that stresses the similarities between the two modes of operation. Section III describes the methods and the samples used in our experiments. Section IV presents the experimental results, and finally, Section $\mathrm{V}$ is devoted to a discussion of practical amplifier design in terms of device parameters.

\section{Theoretical Considerations}

In previous publications [2], [9], [10], the theories of the singly degenerate and the doubly degenerate modes of parametric amplification with externally pumped Josephson junctions were evaluated. A unified description of both modes is, however, straightforward. Since both modes employ a common signal and idler matching circuit, the degeneracy is determined by the relation between signal and pump frequencies. If $f_{s} \sim \frac{1}{2} f_{p}$, the mode becomes singly quasi-degenerate and the principal idler is at $f_{i 1}=f_{p}-f_{s} \sim \frac{1}{2} f_{p}$. If $f_{s} \sim f_{p}$, the mode becomes doubly quasi-degenerate with the idler at $f_{i 2}=$ $2 f_{p}-f_{s}$, i.e., $f_{i 2} \sim f_{s} \sim f_{p}$.

Assuming that the junction is biased at zero dc voltage and is short circuited at all frequencies except in two bands centered at $f_{p}$ and $\frac{1}{2} f_{p}$, the junction voltage contains components only at $f_{s}, f_{p}$, and $f_{i 1}$ or $f_{i 2}$. The Josephson voltage-phase relation

$$
V=\frac{\hbar}{2 e} \dot{\phi}
$$

determines the phase. The voltage and the phase waveforms evidently have the same frequency content except for a dc component $\phi_{0}$ of the phase.

Within the framework of the shunted junction model, the current is determined by

$$
I=\frac{\hbar C}{2 e} \ddot{\phi}+\frac{\hbar}{2 e R} \dot{\phi}+I_{c} \sin \phi .
$$

In the small-signal limit, (2) may be linearized in signal and idler components and a Fourier analysis of the current leads to the small-signal admittance matrix $\vec{Y}=\left\{Y_{n m}\right\}$

$$
\begin{aligned}
\left(\begin{array}{c}
I_{s} \\
I_{i 1}^{*} \\
I_{i 2}^{*}
\end{array}\right)= & \left(\begin{array}{lll}
1+j \delta_{s} / \xi_{s} & -J_{1} \sin \phi_{0} / \xi_{i 1} & j J_{2} \cos \phi_{0} / \xi_{i 2} \\
-J_{1} \sin \phi_{0} / \xi_{s} & 1-j \delta_{i 1} / \xi_{i 1} & -J_{1} \sin \phi_{0} / \xi_{i 2} \\
-j J_{2} \cos \phi_{0} / \xi_{s} & J_{1} \sin \phi_{0} / \xi_{i 1} & 1-j \delta_{i 2} / \xi_{i 2}
\end{array}\right) \\
& \cdot\left(\begin{array}{l}
V_{s} \\
V_{i 1}^{*} \\
V_{i 2}^{*}
\end{array}\right)
\end{aligned}
$$

where currents and voltages are normalized to $I_{c}$ and $R I_{c}$, respectively. The frequencies $\xi_{n}$ are normalized to $\omega_{c}=$ $2 e R I_{c} / \hbar$. The argument of the Bessel function $J_{n}$ is $\alpha=$ $2 e V_{p} / \hbar \omega_{p}$, where $V_{p}$ is the pump-induced voltage. The quantity $\delta_{n}$ is the plasma resonance term $\delta_{n} \equiv \beta \xi_{n}^{2}-J_{0} \cos \phi_{0}$, where $\beta=\omega_{c} R C$ is the McCumber parameter.

In the present simplified discussion, we omit all details concerning the practical realization of the signal-matching circuit. We assume the idealized situation when the input/output line in the passband of the matching circuit is described by a characteristic impedance $Z_{0}$, and outside the passband it shortcircuits the junction. The junction input admittance becomes

$$
Y_{\text {in }}=Y_{11}-\frac{Y_{1 m} Y_{m 1}}{g+Y_{m m}}, \quad g=R / Z_{0}
$$

with $m=2$ or $m=3$ for the SDA or DDA, respectively. The signal gain is found from $G=\left|g-Y_{\text {in }}\right|^{2} /\left|g+Y_{\text {in }}\right|^{2}$ and may, at center band $\left(\xi \equiv \xi_{s} \simeq \xi_{i 1}\right.$ or $\left.\xi_{s} \simeq \xi_{i 2}\right)$, be written as

$$
G=\frac{\left(\xi^{2}\left(1-g^{2}\right)+\delta^{2}-\gamma^{2}\right)^{2}+(2 \xi g \delta)^{2}}{\left(\xi^{2}(1+g)^{2}+\delta^{2}-\gamma^{2}\right)^{2}}
$$

where $\gamma=J_{1} \sin \phi_{0}$ for the SDA and $J_{2} \cos \phi_{0}$ for the DDA.

In both modes, the operating point must be chosen such that the denominator of (5) is near zero, i.e.,

$$
\gamma^{2} \simeq \xi^{2}(1+g)^{2}+\delta^{2} .
$$

The high-gain condition (6) imposes several restrictions on the amplifier parameters. Since $\gamma$ (proportional to $J_{1}$ or $J_{2}$ ) is limited, it is, in both modes, advantageous to tune $\delta=\beta \xi^{2}$ $J_{0} \cos \phi_{0}$ to zero. If $\xi(1+g)$ approaches the maximum value of $J_{1}$ or $J_{2}$, it becomes mandatory. In the case of a negligible junction capacitance $(\beta \rightarrow 0)$, high gain is most conveniently achieved operating near the first zero of $J_{0}$, where both $J_{1}$ and $J_{2}$ are almost at their maximum. 
In all experiments in the DDA mode [1] -[5], the device has been operated near the first zero of $J_{0}(\alpha)$ and at zero dc current bias $\left(\cos \phi_{0}=1\right)$ in order to maximize $\gamma$. No attempt has been made to take advantage of the internal plasma resonance. In the experiments on the SDA mode reported in the subsequent sections, the plasma resonance is used to resonate out the average Josephson inductance (the term $J_{0} \cos \phi_{0}$ ). This is most conveniently done by means of the dc-bias current, since $\gamma=J_{1} \sin \phi_{0}$ increases with increasing bias while $J_{0} \cos \phi_{0}$ decreases. The SDA may be parametrically active at pumppower levels where $J_{0}$ has decreased by only a few percent. An important quantity characterizing the performance of the amplifier is the gain-bandwidth product. To conclude the theoretical section, we state a simple general expression valid in the high-gain limit, provided that the plasma resonance condition $\delta=0$ is also satisfied. For both modes the result is

$$
G^{1 / 2} \frac{\Delta \omega}{\omega}=\frac{g}{\xi \beta}, \quad \text { with } \xi= \begin{cases}\xi_{p} / 2 & \text { (SDA) } \\ \xi_{p} & \text { (DDA). }\end{cases}
$$

\section{METhODS AND SAMPLES}

\section{A. Samples and Cryogenic Insert}

It is obvious from the discussion in the preceeding section that the sample and matching circuit parameters must be chosen in accord with the particular application.

The samples used in our experiments were $\mathrm{Sn-oxide-Sn} \mathrm{or}$ $\mathrm{Sn}$-oxide- $\mathrm{Pb}$ tunnel junctions. The areas used were typically $50 \mu \mathrm{m} \times 100 \mu \mathrm{m}$ for the $10-\mathrm{GHz}$ samples, $10 \mu \mathrm{m} \times 10 \mu \mathrm{m}$ for the $35-\mathrm{GHz}$ samples, and $10 \mu \mathrm{m} \times 10 \mu \mathrm{m}$ for the $70-\mathrm{GHz}$ samples. The corresponding current densities (at $T=0 \mathrm{~K}$ ) were typically $20 \mathrm{~A} / \mathrm{cm}^{2}, 100-300 \mathrm{~A} / \mathrm{cm}^{2}$, and $500 \mathrm{~A} / \mathrm{cm}^{2}$, giving maximum plasma frequencies of $\sim 25 \mathrm{GHz}, \sim 60-100$ $\mathrm{GHz}$, and $\sim 125 \mathrm{GHz}$, respectively. At $35 \mathrm{GHz}$, both single junctions and 12-junction arrays were investigated. The theoretical discussion was based on a simplified model of the amplifier. In real junctions, stray reactances play an important role. The thin wire of a point contact placed across a waveguide will introduce an inductive load in series with the contact. Also, the series reactance from the short and narrow superconducting strips contacting the junction(s) in thin-film devices are of an inductive nature. In order to compensate the reactance, additional reactive elements are needed, which may impose bandwidth limitation on the amplifier. Hence, (7) of Section II can only estimate the upper limit of the actual bandwidth.

In our experiments, the junctions were mounted across the low-impedance end of three different binomial quarter-wave impedance transformers. The impedance ratio of the $10 . \mathrm{GHz}$ transformer was 188 , the $35-\mathrm{GHz}$ transformer had a ratio of 58 , and the $70-\mathrm{GHz}$ transformer reduced the waveguide impedance by a factor of 64 . The reactive tuning was accomplished by means of an adjustable inductive window followed by a dielectric phase shifter such that the electrical distance between the window and the junction could be varied.

Input and output signals were separated by a room-temperature circulator $(10 \mathrm{GHz})$, a cryogenic circulator $(35 \mathrm{GHz})$, and a room-temperature directional coupler $(70 \mathrm{GHz})$. At $10 \mathrm{GHz}$, the junction was immersed in liquid helium, while in the other cases, the low-temperature part was enclosed in a vacuum can

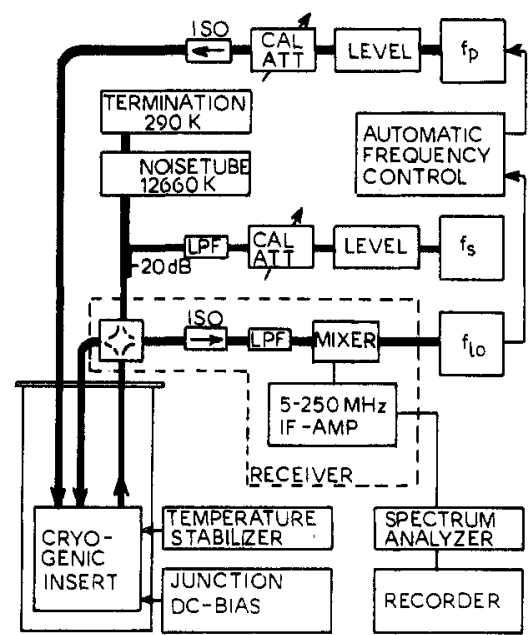

Fig. 1. Block diagram of the experimental setup. The combinations a) $f_{s} \sim 9 \mathrm{GHz}, f_{p} \sim 9 \mathrm{GHz}$, b) $f_{s} \sim 9 \mathrm{GHz}, f_{p} \sim 18 \mathrm{GHz}$, c) $f_{s} \sim 35$ $\mathrm{GHz}, f_{p} \sim 70 \mathrm{GHz}$, and d) $f_{s} \sim 70 \mathrm{GHz}, f_{p} \sim 70 \mathrm{GHz}$ were used.

and the temperature stabilized to within $50 \mu \mathrm{K}$. In all cases, pump power was loosely coupled from the back side of the substrate. This coupling scheme is adequate because of the low pump power needed.

\section{B. The Microwave System}

The microwave receivers were at all three frequencies as shown in Fig. 1. At the lower frequencies, water-cooled Gunn oscillators were used while klystrons were used at $70 \mathrm{GHz}$. The Gunn oscillators (both short- and long-term stability were better than $50 \mathrm{kHz}$ ) were sufficiently stable to be left free running, whereas the klystrons had to be frequency locked to Gunn oscillators. Low-pass filters prevented higher harmonics from reaching the junction and the mixer.

The output from the mixer was amplified by a low-noise $(<2-\mathrm{dB})$ IF amplifier and displayed on a spectrum analyzer (SA) with a resolution bandwidth $B_{\mathrm{SA}}=300 \mathrm{kHz}$. The SA output could be traced on an $X-Y$ recorder.

A waveguide noise tube and a room-temperature termination were used as standards for the noise-temperature measurement.

\section{EXPERIMENTAL RESUltS}

\section{A. $10 \mathrm{GHz}$}

Both the DDA and the SDA were investigated at $10 \mathrm{GHz}$. Only measurements of the gain were performed, as the noise temperatures evidently were very high. This was probably caused by the very low resistance $(0.1-1 \Omega)$ of the junctions, compared to the output impedance of the transformer. This will be discussed in more detail below. The following discussion will refer to a specific junction with $R \sim 1 \Omega$.

In the DDA mode, the gain was only a few decibels. Typical experimental traces are illustrated in Fig. 2. The upper part of the figure shows the $I-V$ curve with and without pump power. It is seen that the pump power needed to produce gain corresponds to a near-complete suppression of the supercurrent. The lower part of the figure shows a spectrum-analyzer display for two different pump-power levels. For trace 1, the pump power is too low to give gain. Only the pump and signal lines are seen above the receiver noise background. Trace 2 shows 


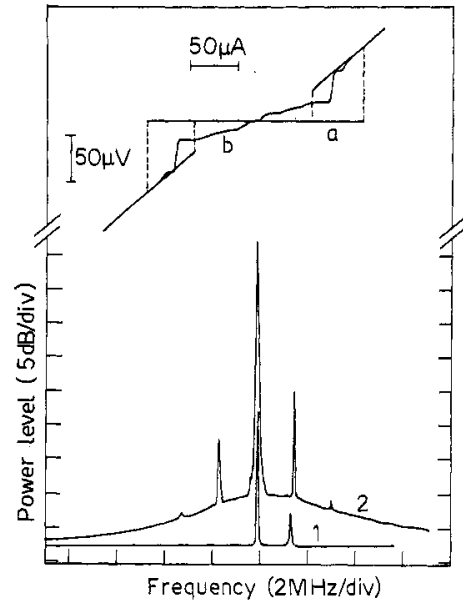

Fig. 2. Doubly degenerate amplification in a single junction. $T=3.59$ $\mathrm{K}, f_{p}=8.5 \mathrm{GHz}$, and $f_{s} \cong 8.5 \mathrm{GHz}$. Junction: $\mathrm{Sn}$-oxide-Sn, $30 \times$ $100(\mu \mathrm{m})^{2}, J_{c}=30 \mathrm{~A} / \mathrm{cm}^{2}(T=0 \mathrm{~K})$. Upper part: $I-V$ curve without $a$ and with $b$ applied pump power. Lower part: 1-reflected signal with low pump power; 2 -with pump power corresponding to $b$ in the upper part.

parametric amplification of a few decibels and the idler at $2 f_{p}-f_{s}$ has appeared. The broad noise rise observed often accompanies the parametric interaction. The bandwidth of the noise rise normally reflects the amplifier bandwidth, indicating here a bandwidth of only a few megahertz. Such small bandwidths are always found in experiments using tunnel junctions-single junctions as well as arrays [3] - and are presumably due to limitations imposed by the embedding microwave circuitry.

The noise rise is not understood in detail. A tentative explanation has been provided by invoking the concept of phase instability [11]. The noise rise, however, is observed in both the DDA and the SDA modes and it is not obvious how the suggested mechanism applies to both cases.

The junction used in Fig. 2 produced substantial gain in the SDA mode [7]. Gains up to $16 \mathrm{~dB}$ in a $4-\mathrm{MHz}$ bandwidth were observed, although they still were associated with noise rise and very-high noise temperatures $(3000 \mathrm{~K})$.

Also in the SDA mode, the relative bandwidth was consistently found less than predicted by theory. Obviously, a more elaborate model of the embedding microwave circuit must be used in order to understand this result $\lceil 10\rceil$.

Fig. 3 illustrates another interesting phenomenon. In both the SDA and DDA modes, amplification is possible on the pump-induced steps at dc voltages $n h f_{p} / 2 e$. The upper part of the figure shows the $I-V$ curve with and without pump power. With the pump applied, steps at $n=1$ and 3 are seen. In the lower part of the figure is shown the SA output with the SA window preset at 1) the IF transpose of the signal frequency and 2) detuned by $200 \mathrm{kHz}$. The output is monitored as a function of bias current on the $n=1$ step. Also shown (the dashed line) is the signal power level at the top of the cryostat. The noise output (trace 2 ) is very high in the region of gain, indicating a high noise temperature. Still, the gain is appreciable $(12 \mathrm{~dB})$. Self-oscillation at half the pump frequency was observed (not shown) on the lower part of the step.

The occurance of self-oscillations indicates that the infinite

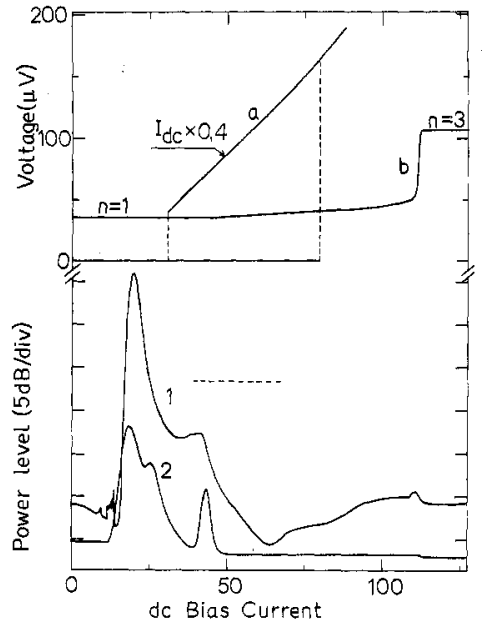

Fig. 3. Singly degenerate parametric amplification on the $n=1 \mathrm{RF}$ induced step. $T=3.50 \mathrm{~K}, f_{p}=17.0 \mathrm{GHz}$, and $f_{s}=8.57 \mathrm{GHz}$. The same junction as in Fig. 2. Upper part: $I-V$ curve without $a$ and with $b$ applied pump power (note the different current scales). Lower part: Received power at frequencies $1-f_{s}$ and $2-f_{s}+200 \mathrm{kHz}$. The dashed line shows input signal level.

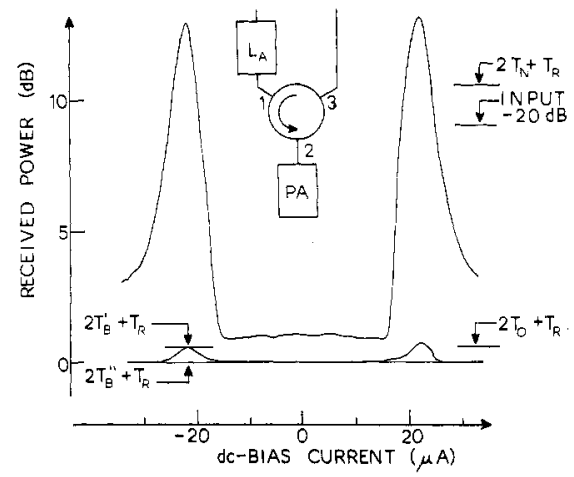

Fig. 4. Received power versus dc bias currents at $f_{s}$ (upper curve) and $f_{s}+1 \mathrm{MHz}$ (lower curve) with $f_{s}=36.15 \mathrm{GHz}, f_{p}=72.31 \mathrm{GHz}$, and $T=2.96 \mathrm{~K}$. The indications on the right-hand side are the recorded $Y$ deflections used for gain and noise temperature calibration. $T_{0}$, $T_{N}$, and $T_{R}$ are equivalent noise temperatures of the room-temperature termination, the ignited noise tube, and the receiver, respectively. The inset shows a sketch of the temperature-stabilized part of the experimental setup.

gain condition (6) has been satisfied. In practice, this is a useful diagnostic tool in the search for parametric effects [12].

\section{B. $35 \mathrm{GHz}$}

1) Single Junctions, SDA Mode: At $35 \mathrm{GHz}$, measurements were performed on both single junctions and arrays. The best result was obtained on a $10.4-\Omega$ single junction where a maximum gain of $11.6 \mathrm{~dB}$ referred to the amplifier port of the cooled circulator was achieved. The corresponding bandwidth was of order $10 \mathrm{MHz}$ and the SSB noise temperature was 400 K. At lower junction resistances $(\sim 1-2 \Omega)$, reasonably high gain was still obtained, but with a much degraded noise performance.

A typical experimental trace for the $10.4-\Omega$ junction is shown in Fig. 4. Here is plotted the received power versus dc-bias current at the signal frequency (upper curve) and 1 $\mathrm{MHz}$ away (lower curve). Shown to the right are the recorded 


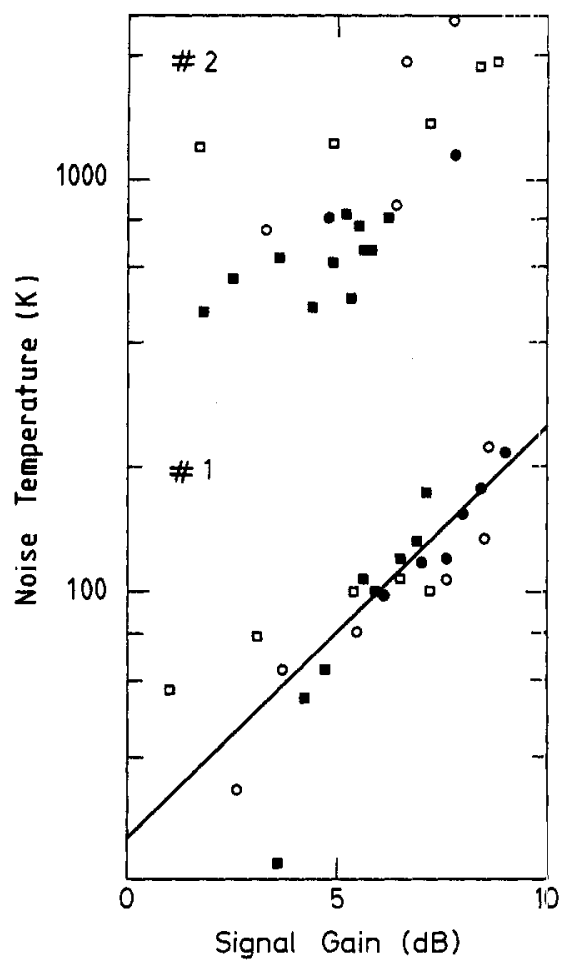

Fig. 5. Noise temperature versus gain as measured by varying only one parameter at a time. Different symbols are used for each prarameter: dc bias current (open squares), signal frequency (full squares), pu:mp power (open circles), and temperature (full circles). $1-R_{N}=10.4 \Omega$. $2-R_{N}=1.5 \Omega$.

$Y$ deflections used for calculating the gain of the parametric amplifier and the noise temperature of the receiver. Once these parameters are known, the intrinsic noise temperature of the parametric amplifier can be calculated from the noisebackground curve by taking into account all noise contributions that fall within the receiver sidebands. The maximum gain found here is $7.2 \mathrm{~dB}$ in a $20 \mathrm{-MHz}$ bandwidth, and an SSB noise temperature of $100 \pm 25 \mathrm{~K}$. Extensive measurements were performed on this junction. One surprising, result was that the gain and noise temperature increased together (in contrast to what is found for varactor parametric amplifiers), a correlation rather independent of the parameter by rneans of which the gain was changed. Fig. 5 also includes results obtained for a $1.5-\Omega$ junction, illustrating the same general behavior, but with much higher noise temperatures. The stability of the amplifier against changes in bias conditions were studied. A typical measure of the gain dependence on pump power is $\Delta G / \Delta P \sim 3 \mathrm{~dB} / 1 \mathrm{~dB}$. As shown in Fig. 4, the amplifier can be conveniently tuned by changing the dc-bias current at constant temperature. However, since the plasmal frequency is proportional to $I_{c}^{1 / 2}$, the temperature dependencs of $I_{c}$ may cause both gain and noise temperature to be sensitive to temperature variations. In the case of the 10.4- $\Omega$ junction (see Fig. 5) we observed that a $20 \mathrm{mK}$ change in temperature produced a 3- $\mathrm{dB}$ change in both gain (from 6 to $9 \mathrm{~dB}$ ) and noise temperature (from 100 to $200 \mathrm{~K}$ ). In conclusion, the stability of the amplifier is sufficiently good for practical applications.

The correlation between gain and noise temperature observed here is also found in the DDA mode [3], as noted by Feldman and Levinsen [13]. They ascribe the apparent proportionality

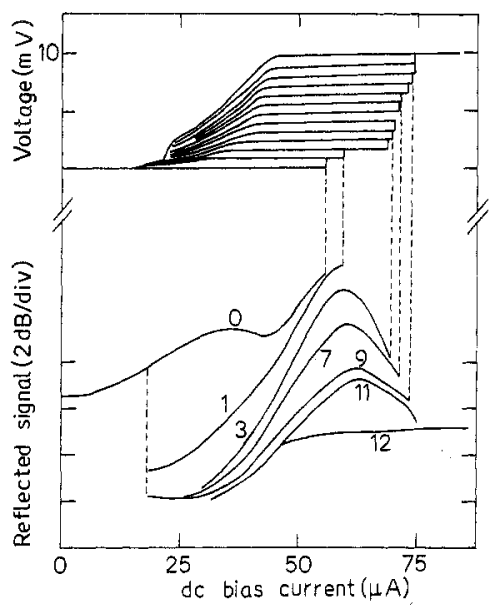

Fig. 6. $I-V$ curve for a 12-junction array of $10 \times 10 \mu \mathrm{m}^{2} \mathrm{Sn}$-oxide-Sn junctions (upper part). Reflected power at $34.0 \mathrm{GHz}$ (lower part). The traces are labeled by the number of junctions that have switched to the finite voltage state. The maxima correspond to plasma resonance excitations. $T=2.88 \mathrm{~K}$.

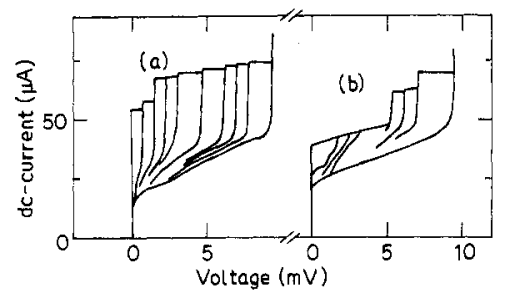

Fig. 7. I-V curves for the array in Fig. $6(a)$ without applied pump power, $(b)$ with pump power applied at $68 \mathrm{GHz}$.

between noise temperature and gain to an operating-point instability which may cause large excursions from the stable limit cycle when the total noise power becomes appreciable compared to the pump power.

2) Arrays, $S D A$ Mode: Preliminary measurements on arrays in the SDA mode were not very encouraging. Gains higher than those achieved with a single junction were never obtained and the noise temperatures were much higher. At very-low power levels, the junctions were rather identical, as inferred from the measured supercurrents. For a typical array, the spread in supercurrents was of the order of 3 percent for ten of the junctions, whereas the remaining two had 20 percent smaller supercurrents. This is illustrated in Fig. 6, which shows the result of a small-signal reflection measurement. Two resonances are seen, one corresponding to the two junctions with low supercurrents and one corresponding to the other ten junctions. The numbering of the curves tells how many junctions have switched to the finite-voltage state. Fig. 7 shows that the spread in supercurrents is drastically enhanced, as the pump power is increased. The plasma resonances of the individual junctions are, therefore, detuned by the pump. Hence, the problem of using arrays is twofold. First, any small differences in junction parameters are magnified by the pump, and second, the parametrically active junctions will be loaded in some unknown way by the nonparticipating junctions. The best operating point found in the experiments on this array had seven junctions switched. The gain was comparable to that of single-junction devices. The 


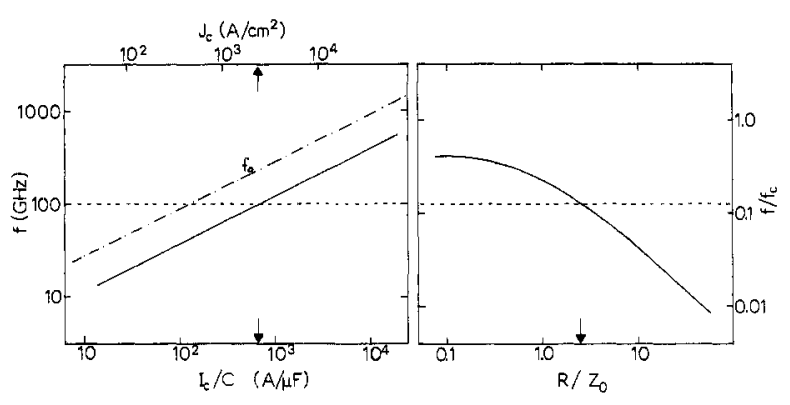

(a)

(b)

Fig. 8. Dimensioning of a Josephson-tunnel-junction parametric amplifier. The frequency and current-density scales are appropriate for lead. The curves are discussed in the text.

amplifier was, however, very unstable and had a rather high noise temperature.

\section{C. $70 \mathrm{GHz}$}

The signal was coupled into the cryostat using an oversize 35-GHz waveguide with a tapered transition to the $70-\mathrm{GHz}$ quarter-wave transformer. Only the DDA mode was investigated at this frequency. Even though parametric effects with noise rise and the appearance of an idler were observed, no net gain was achieved.

\section{The Choice of Junction Parameters}

The important criterion in the choice of junction parameters is the high-gain condition (6) which may be written as

$$
\begin{aligned}
& \left(\frac{\omega_{s}}{\omega_{c}}\right)^{2}\left(1+R / Z_{0}\right)^{2}+\left(J_{0} \cos \phi_{0}-\left(\frac{\omega_{s}}{\omega_{0}}\right)^{2}\right)^{2} \\
& =\left\{\begin{array}{l}
\left(J_{1} \sin \phi_{0}\right)^{2}(\mathrm{SDA}) \\
\left(J_{2} \cos \phi_{0}\right)^{2} \text { (DDA) }
\end{array}\right.
\end{aligned}
$$

where the maximum plasma frequency $\omega_{0}=\left(2 e I_{c} / \hbar C\right)^{1 / 2}$ has been introduced.

From (8), it follows that the highest signal frequency attainable is found at plasma resonance and for $R / Z_{0} \rightarrow 0$. The limiting frequency is $\omega_{s} \sim 0.58 \omega_{c}$ and $\omega_{s} \sim 0.49 \omega_{c}$ for the SDA and the DDA, respectively. In order to satisfy the resonance condition we must have $\omega_{s} \lesssim \omega_{0}$. Since $\omega_{c}$ is proportional to the $R I_{c}$ product, a high operating frequency requires high $T_{c}$ materials. Also, the plasma frequency must be high, implying that the ratio $I_{c} / C$ should be large. This calls for a high current-density junction and a low dielectric constant. In contrast to the high current-density requirement, it has been found $[10],[14]$ that optimum noise performance is achieved if $R>Z_{0}$. This underscores the necessity for small-area junctions and impedance transformation. Furthermore, the gainbandwidth product increases with decreasing area (decreasing capacitance).

The choice of optimum-junction parameters can now be made with the aid of Fig. 8. Fig. 8(a) has $I_{c} / C$ and the frequency $f$ as parameters. The plasma frequency is represented by the dash-dot line of slope $\frac{1}{2}$. In Fig. $8(\mathrm{~b})$, the abscissa is $R / Z_{0}$ and the ordinate is the reduced frequency $f / f_{c}$ (righthand scale). In this parameter space, curves of infinite gain (ING curves) can be drawn. Stable amplification is found in the region just above the $I N G$ curve. The $I N G$ curve, however, is not uniquely determined as can be seen from (8). The operating point may be chosen to achieve either plasma resonance, maximum stability against pump-power fluctuations, or maximum stability against dc-bias fluctuations. Independent of the choice, the best operating point is always found below the plasma-resonance line denoted $f_{0}$ in Fig. 8(a), and just above the ING curve in Fig. 8(b). As the pump power is increased from zero, the ING curve shifts towards higher reduced frequencies. At the same time the plasma frequency decreases. Also, a change in dc-bias current will shift the $I N G$ curve and the plasma frequency.

To illustrate the design procedure, let us consider as an example a $100-\mathrm{GHz}$ singly degenerate parametric amplifier. The selection of a $\mathrm{Pb}$-oxide- $\mathrm{Pb}$ junction $\left(f_{c} \sim 800 \mathrm{GHz}\right.$ at $T=0$ K) gives a sufficiently low reduced-signal frequency (the dashed line in Fig. 8(a) and (b)). The capacitance per unit area is $\sim 3.5 \mu \mathrm{F} / \mathrm{cm}^{2}$ for $\mathrm{Pb}$-based junctions [15] rather independent of current density in the range corresponding to plasma frequencies between 10 and $200 \mathrm{GHz}$. Using this value, the current-density axis also shown in Fig. 8(a) can be drawn.

The determination of $R / Z_{0}$ completes the design. To maximize the gain-bandwidth product and optimize the noise performance, $R / Z_{0}$ should be as large as possible. This obviously requires that the $I N G$ curve is pushed towards high frequencies. Consequently, both $J_{1}(\alpha)$ and $\sin \phi_{0}$ should be maximized by adjusting the pump power and the bias current. However, bias points close to the maximum supercurrent are unstable. A reasonable value is $\sin \phi_{0}=0.8$. This unambiguously determines the ING curve shown in Fig. 8(b) and the value of $R / Z_{0} \sim 2.5$. At this operating point, the plasma frequency is given by $\omega_{0}\left(J_{0} \cos \phi_{0}\right)^{1 / 2} \sim 0.45 \omega_{0}$, which corresponds to the dashed line in Fig. 8(a).

From the condition that the signal frequency should coincide with the plasma frequency, we find the necessary current density to be $J_{c} \simeq 2400 \mathrm{~A} / \mathrm{cm}^{2}$, which is readily achieved with a $\mathrm{Pb}$ junction. Assuming further a load resistance $Z_{0}$ (transformed waveguide impedance) of $3 \Omega$, we can calculate the junction dimensions to be $3 \mu \mathrm{m} \times 3 \mu \mathrm{m}$. Such junctions are readily manufactured using standard photolithographic techniques. Using the numbers previously determined, the gainbandwidth product may be calculated from (7). The result is $G^{1 / 2} \Delta \omega / \omega=1.7$. An estimate of the noise temperature may also be made in the high-gain limit. Using [10], we find $T_{\text {noise }} \cong 8 \mathrm{~K}$. This result neglects quantum corrections. However, the quantum limit is $T_{\text {noise }}=\hbar f / k \ln 2$, which at $f=100$ $\mathrm{GHz}$ gives $T_{\text {noise }}=6.9 \mathrm{~K}$. Consequently, quantum effects cannot be neglected in a consistent noise theory. This would, however, require a more elaborate model than the simple, shunted-junction model commonly used, as this model breaks down at the high frequencies where the quantum corrections become important.

\section{CONCLUSION}

The principles of the operation of Josephson-junction amplifiers have been discussed and illustrated by experimental results. The similarities between the two modes, SDA and DDA, have been stressed. 
The experimental results obtained so far have been encouraging. It has become obvious that the coupling circuit plays a central role. Josephson-junction devices are not easily incorporated in waveguide structures, and improved performance may possibly be achieved by the use of low-impedance stripline techniques. Preliminary measurements on microbridges in microstrip structures seem to favor this approach.

Based on simplifying assumptions, a guideline for the specification of junction parameters has been worked out. The design of a $100-\mathrm{GHz}$ amplifier was carried out as an example, and according to the theoretical predictions, operation with lownoise temperature and a reasonable gain-bandwidth product should be feasible.

\section{REFERENCES}

[1] P. T. Parrish, M. J. Feldman, H. Ohta, and R. Y. Chiao, "Four photon parametric amplification," Rev. Phys. Appl., vol. 9, pp. 229-232, 1974.

[2] M. J. Feldman, P. T. Parrish, and R. Y. Chiao, "Parametric amplification by unbiased Josephson junctions," J. Appl. Phys., vol. 46, pp. 4031-4042, 1975.

[3] S. Wahisten, S. Rudner, and T. Claeson, "Arrays of Josephson tunnel junctions as parametric amplifiers," J. Appl. Phys., vol. 49, pp. 4248-4263, 1978.

[4] Y. Taur and P. L. Richards, "Parametric amplification and oscillation at $36 \mathrm{GHz}$ using a point contact Josephson junction," $J$. Appl. Phys., vol. 48, pp. 1321-1326, 1977.

[5] F. Goodall, F. Bale, S. Rudner, T. Claeson, and T. F. Finnegan, "Parametric amplification in Josephson tunnel junction arrays at
33 GHz," IEEE Trans. Magn., vol. MAG-15, pp. 458-461, 1979.

[6] N. F. Pedersen, M. R. Samuelsen, and K. Saermark, "Parametric excitation of plasma oscillations in Josephson junctions," $J$. Appl. Phys., vol. 44, pp. 5120-5124, 1973.

[7] J. Mygind, N. F. Pedersen, and O. H. Soerensen, "X-band singly degenerate parametric amplification in a Josephson tunnel junction," Appl. Phys. Lett., vol. 32, pp. 70-72, 1978.

[8] J. Mygind, N. F. Pedersen, O. H. Soerensen, B. Dueholm, and M. T. Levinsen, "Low noise parametric amplification at $35 \mathrm{GHz}$ in a single Josephson tunnel junction," Appl. Phys. Lett., vol. 35 , pp. 91-93, 1979.

[9] M. J. Feldman, "The thermally saturated SUPARAMP," J. Appl. Phys., vol. 48, pp. 1301-1310, 1977.

[10] O. H. Soerensen, B. Dueholm, J. Mygind, and N. F. Pedersen, "Theory of the singly quasi-degenerate Josephson junction parametric amplifier," J. Appl. Phys., to be published.

[11] R. Y. Chiao, M. J. Feldman, D. W. Peterson, B. A. Tucker, and M. T. Levinsen, "Phase instability noise in Josephson junctions," in Future Trends in Superconductive Electronics. (AIP Conf. Proc.), vol. 44, pp. 259-263, 1978.

[12] N. F. Pedersen, O. H. Soerensen, B. Dueholm, and J. Mygind, "Half-harmonic parametric oscillations in Josephson junctions," J. Low Temp. Phys., vol. 38, nos. 1/2, pp. 1-23, 1980.

[13] M. J. Feldman and M. T. Levinsen, "Gain-dependent noise temperature of Josephson parametric amplifiers," Appl. Phys. Lett., in print.

[14] D. W. Peterson, "The unclamped and current clamped SUPARAMP: Studies of unbiased Josephson junction parametric amplifier," Ph.D. dissertation, University of California, Berkeley, CA, 1978.

[15] J. M. Eldridge and J. Matisoo, "Measurement of tunnel current density in metal-oxide-metal system as a function of oxide thickness," in Proc. LT 12. Tokyo, Japan: Academic Press of Japan, 1971, pp. 427-428.

\title{
Microwave Theory of Josephson Oscillators
}

\author{
CHARLES V. STANCAMPIANO, MEMBER, IEEE
}

\begin{abstract}
In this paper, we deal with a model of a specific Josephson microwave circuit, that of a Josephson oscillator, and show that the RF behavior of a real Josephson oscillator may be predicted from a knowledge of the experimentally measured microwave circuit parameters, the junction critical current, and junction shunt resistance. Based on observations made with an electronic analog, we present an approximate analy tical method for calculating the junction impedance or, rigorously speaking, the appropriate single sinusoidal-input describing function. Emphasis is placed on the proper use of the impedance, for example, in
\end{abstract}

Manuscript received February 12, 1980; revised June 12, 1980. This work was supported by the Office of Naval Research under Contract N00014-76-C-0001 with the Center for Naval Analyses of the University of Rochester.

The author is with the Department of Electrical Engineering, University of Rochester, Rochester, NY 14627. calculating the operating point and the expected output power of the oscillator. The circuit model used is that of a junction, described by the resistively shunted junction model, coupled to a series $L C R$ resonance. Further confirmation of the validity of the circuit-theory approach is obtained by using the injection-locked oscillator theory of Kurokawa to predict the in-lock amplitude variation of a Josephson oscillator exposed to a weak synchronizing signal.

Experimental data describing the amplitude variation and output power of an oscillator consisting of a point-contact junction placed in a $9.72-\mathrm{GHz}$ coaxial resonator are presented. The data demonstrate the reasonable agreement obtained when the measured critical current and shunt resistance are used with the analytical expression for the junction impedance and the circuit theory to predict the RF behavior of a Josephson oscillator.

Circuits more complex than our specific example may be handled by means of describing function techniques recently developed in the area of nonlinear solid-state microwave devices. 